\title{
Modalités de production de bœufs «laitiers» de 30 mois à l'herbe
}

\author{
D Dozias ${ }^{1}, \mathrm{D}$ Micol 2, JR Peccatte 1 \\ ${ }^{1}$ INRA, service d'expérimentation animale, 61310 Exmes; \\ 2 INRA-Theix, laboratoire d'adaptation des herbivores aux milieux, \\ 63122 Saint-Genès-Champanelle, France
}

(Reçu le 13 septembre 1993 ; accepté le 21 septembre 1994)

\begin{abstract}
Résumé - Nous avons précisé les effets de différentes modalités d'élevage sur les performances de production de bœufs de 30 mois de génotypes laitiers finis à l'herbe. Les premières comparaisons ont porté sur la période du jeune âge. Elles ont permis de tester l'influence du pâturage avant un an en interaction avec la nature du régime hivernal (à base d'ensilage d'herbe ou de maïs) autour de 1 an, puis du niveau alimentaire avant 14 mois. Ensuite, nous avons étudié les effets des variations du niveau alimentaire par la succession de périodes de restriction alimentaire en stabulation et de périodes d'alimentation libérale au pâturage. La pratique du pâturage avant l'âge de 1 an facilite la transition lors de la mise à l'herbe vers 14 mois. Un niveau alimentaire haut avant 9 mois permet une croissance élevée. Dans ces 2 cas l'avantage de poids acquis est conservé et permet de raccourcir la durée de la période de finition. Les effets de restrictions alimentaires entre 9 et 14 mois sont compensés par une croissance supérieure lors de la phase de pâturage ultérieure. En revanche, une restriction entre 21 et 26 mois occasionne un allongement de la période de finition. Dans tous les cas, les animaux ayant bénéficié du niveau alimentaire le plus élevé sont significativement plus gras. Au total, 275 bœufs ont été abattus à un poids vif moyen de $707 \mathrm{~kg} \pm 43$. Ils ont produit des carcasses pesant $375 \mathrm{~kg} \pm 27$ en moyenne et comprenant $65,7 \% \pm 2,1$ de muscles et $17,7 \% \pm 2,2$ de tissus adipeux.
\end{abstract}

bœuf / croissance / carcasse / modalités d'élevage / croissance compensatrice / pâturage

Summary - Mode of management for 30-month-old steers in dairy breeds. The effects of several modes of cattle management were tested on the increase of production characteristics in 30-monthold steers from Normandy, fattened on grass, using dairy breed steers (Friesian and Normand). The first trial evaluated the effects of pasture grazing on steers before they reached 1 year of age, and the type of feed (maize or grass silage) consumed during the winter, when the animals were brought indoors, between 9 and 14 months of age. In the 2nd trial, the 2 growth rates between 4 and 14 months were compared. Here, the effect of succeeding periods of nutrition restriction during winter and high feed supply in pasture were studied. In trial 3, the restriction period occurred between 21 and 26 months. In the 4th trial, there were 2 periods of restriction, between 9 and 14 months and between 21 and 26 months. The measurements of the live weights and growth rates were recorded (table III). The body and carcass composition at slaughter were also measured (tables IV and V). A total of 275 
steers were slaughtered at $707 \mathrm{~kg}$ live weight. They had a mean carcass weight of $375 \mathrm{~kg}$. Their carcasses were analysed to be $65.7 \%$ lean and $17.7 \%$ fat. Steers that experienced pasture grazing before 1 year of age had an advantage over the steers at the transition at the 2nd grazing at 14 months (fig 1). On d 15, these steers were $25 \mathrm{~kg}$ (live weight) heavier and this advantage was conserved until slaughter, where they had the same carcass composition after a $38 d$ shorter fattening period. Thus, steers that had grazed before attaining 1 year of age had a better adaptability at the next grazing period at 14 months. The nature of the silage used during the winter between 9 and 14 months had no effect on the subsequent growth and eventual carcass composition. In the $2 n d$ trial, steers that had a high level of growth before reaching 9 months of age had a subsequent advantage which was conserved and permitted a shorter fattening period. A good growth compensation was observed during the following pasture period for the effects of the restriction period occurring between 9 and 14 months. After the restriction between 21 and 26 months, the duration of fattening increased (22 d in the 3rd trial) (fig 2). In all of the trials, the carcasses of the restricted steers were less fat. After a period of restriction, all the animals showed an increased growth rate. This increase varied with the age of the steers during the restriction period. If it occurred before the animals had obtained 9 months of age, there was poor growth compensation. After this age, however, the growth compensation was complete, and there was even an increase in fattening for the period of restriction between 21 and 26 months. A restriction period that occurs in winter after the animals have reached 9 months of age or a small level of growth before 9 months permits economizing on expensive forage and allows for a better use of pasture. However, high forage diets permit easier slaughtering of the beef. The level of growth in winter is chosen in relation to the duration of the pasture period of fattening.

steer / growth / carcass / mode of production / compensatory growth / grazing

\section{INTRODUCTION}

Dans les conditions françaises, la production de bœufs de 30 mois décrite par Micol (1986) permet, à partir de veaux d'origine laitière nés en hiver, de produire des carcasses de 350 à $400 \mathrm{~kg}$ satisfaisant les besoins du marché et de répondre aux contraintes de la gestion de l'espace rural (occupation des sols, limitation des pollutions...). En effet, ces animaux ne sont pas conduits pour atteindre en permanence leur potentiel de croissance, mais pour valoriser au mieux les ressources herbagères. Ainsi, l'essentiel du gain de poids est réalisé au pâturage, les niveaux de croissance en stabulation restant modérés. Les animaux nés en hiver sont sevrés vers 3 mois, audelà de $100 \mathrm{~kg}$. La mise à l'herbe a lieu, souvent pour la première fois, au printemps vers 14 mois. Le pâturage peut se prolonger jusqu'en novembre si les conditions pédo-climatiques le permettent. Après une nouvelle période de stabulation, les animaux sont finis à l'herbe entre juillet et octobre, selon les conditions fourragères et la précocité du génotype de l'animal.

Cette production traditionnelle a été remplacée progressivement par celle de jeunes bovins mâles et diminue rapidement, le nombre de têtes abattues ayant été divisé par 2 en 10 ans selon I'OFIVAL (1991). C'est pourquoi un programme de recherche sur l'optimisation technique de ces productions a été engagée depuis 1982 par l'INRA au domaine du Pin-au-Haras en Normandie (61).

L'influence des facteurs d'élevage, tels que la nature de l'alimentation ou la forme de la courbe de croissance, dans le jeune âge (avant 14 mois), sur les performances de production de bœufs finis à l'herbe à 30 mois, est moins connue que dans le cas des jeunes bovins abattus à 18-24 mois (Berge, 1991; Berge et al, 1991). Ainsi, la mise à l'herbe des veaux ne se fait pas systématiquement l'année de leur naissance, bien que la sortie au pâturage paraisse souhaitable car elle permet d'acquérir très tôt l'aptitude au pâturage (Giovanni, 1982). De 
même, s'il est bien connu que tout retard de croissance avant 9 mois semble acquis, les animaux développant peu de capacité de croissance compensatrice à cet âge (Micol, 1986), le niveau de croissance lors des périodes suivantes mérite d'être mieux précisé, notamment pour obtenir des carcasses suffisamment lourdes vers 30 mois.

L'utilisation de la croissance compensatrice des bovins permet une exploitation maximale de l'herbe par le pâturage, des retards de croissance, provoqués par un faible niveau alimentaire en stabulation durant l'hiver, étant comblés au cours de la saison de pâturage suivante. Les conséquences de ces croissances à courbe discontinue au cours de la vie de l'animal demandent à être précisées.

Aussi avons nous étudié l'influence du pâturage précoce et de la nature du régime hivernal avant 14 mois. Ensuite, nous avons cherché à situer le niveau optimal de complémentation dans le jeune âge. Ces modalités de conduite étant définies, nous avons précisé l'impact de la forme de la courbe de croissance. Dans un premier temps, nous avons induit des écarts de niveaux de croissance au cours de l'hiver entre 21 et 26 mois. Ensuite ces écarts de niveaux de croissance ont été répétés au cours des 2 périodes hivernales successives entre 9 et 14 mois puis entre 21 et 26 mois.

L'étude a, au total, duré 8 années et portés sur 275 bœufs d'origine laitière, élevés et suivis pendant l'intégralité de leur cycle de production.

\section{CONDITIONS EXPÉRIMENTALES}

\section{Milieu naturel}

Le domaine INRA du Pin-au-Haras est situé en Normandie (61) (latitude $49^{\circ} \mathrm{N}$, longitude $0^{\circ} \mathrm{E}$, $200 \mathrm{~m}$ d'altitude). Les sols limono-argileux sont sensibles aux déséquilibres hydriques. Le climat, de type océanique, entraîne une pluviométrie relativement bien répartie tout au long de l'année. En moyenne, depuis 30 ans, les précipitations annuelles sont de $709 \mathrm{~mm}$ réparties sur 161 j. La proximité de la mer (60 km à vol d'oiseau) maintient en permanence une humidité élevée et des températures assez douces. Janvier est le mois le plus froid avec $2,8^{\circ} \mathrm{C}$ de température moyenne et juillet le plus chaud avec $16,2^{\circ} \mathrm{C}$.

Ces caractéristiques pédo-climatiques favorables expliquent la présence importante de prairies de longue durée. De fait, l'agriculture régionale est basée sur l'exploitation de l'herbe par des bovins (lait et viande).

\section{Modalités expérimentales}

Six séries d'animaux d'un poids moyen d'environ $60 \mathrm{~kg}$ ont été introduites sur le domaine pour y être suivies expérimentalement jusqu'à leur départ à l'abattoir à 30 mois après une finition à l'herbe. Les animaux ont été répartis dans un schéma expérimental combinant 1 ou 2 facteurs de variation (tableau I) selon les essais.

\section{Essai 1}

Le schéma expérimental combine 2 facteurs de variation portant sur la nature de la ration pour un même niveau de croissance des animaux.

Le premier facteur étudié oppose l'utilisation du pâturage (traitement PAT) à l'alimentation à l'auge en stabulation (traitement STA) entre 4 et 9 mois. Le deuxième facteur expérimental est le type d'aliment de la ration hivernale entre 9 et 14 mois : ensilage de mais (traitement EM) et ensilage d'herbe (traitement EH). Dans tous les traitements, les animaux sont conduits de façon à avoir une croissance identique au cours des 2 périodes d'application des traitements ( $1000 \mathrm{~g} / \mathrm{j}$ durant la période correspondant au pâturage du traitement PAT ; $900 \mathrm{~g} / \mathrm{j}$ pendant la période hivernale en stabulation entre 9 et 14 mois).

Quel que soit le traitement antérieur appliqué, tous les animaux ont été mis à l'herbe à 14 mois, sans transition alimentaire afin d'exacerber d'éventuelles différences d'adaptation au pâturage.

L'essai a concerné 2 séries d'animaux. Ceuxci ont été allotés entre les 2 traitements PAT et STA juste avant la mise à l'herbe du lot PAT. Au moment du retour en stabulation du lot PAT, chaque lot a été subdivisé en 2 , selon la modalité $\mathrm{EM}$ ou EH de l'alimentation hivernale. 
Tableau I. Modalités expérimentales et conduite des essais.

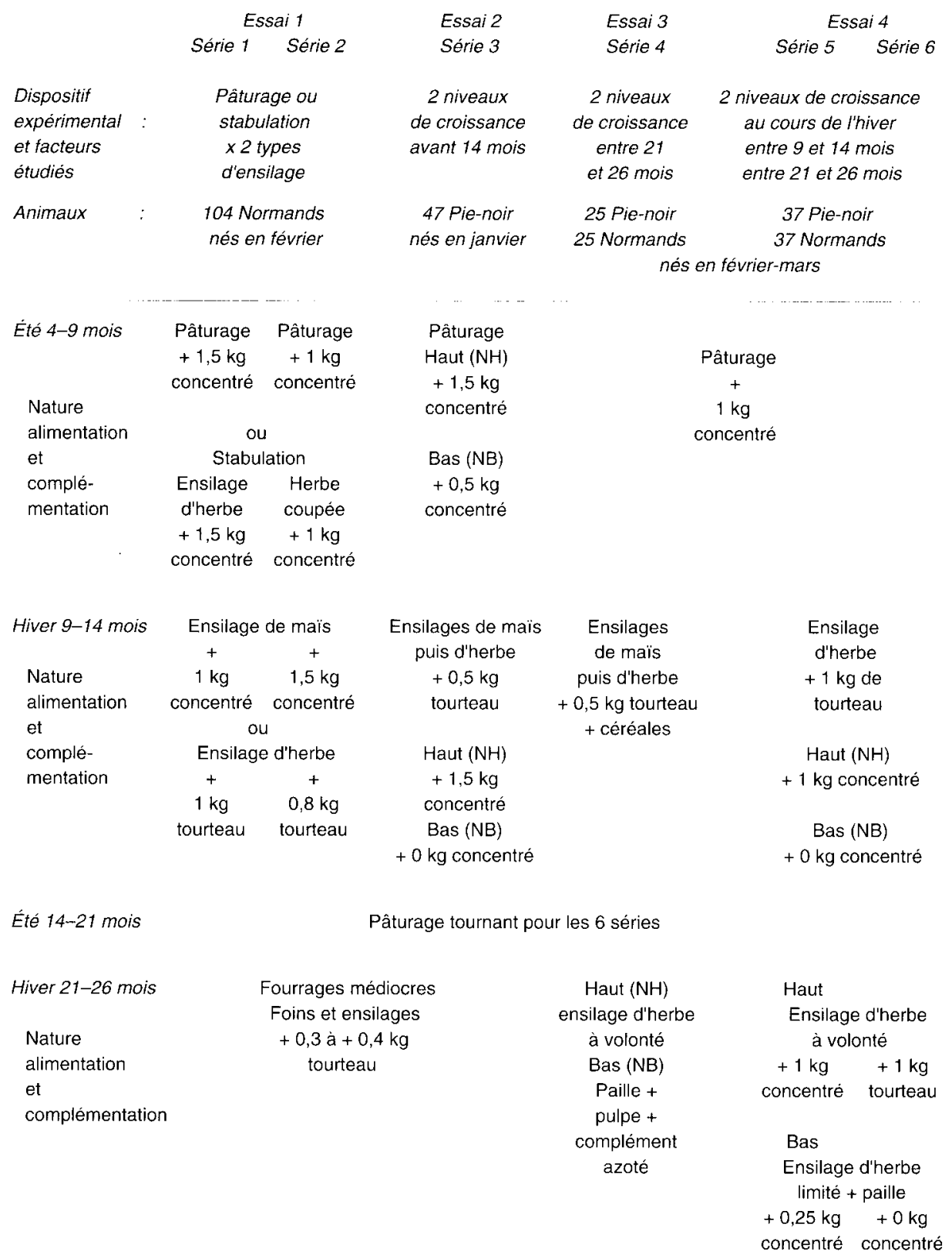




\section{Essai 2}

Le facteur étudié est le niveau de croissance induit par le niveau d'alimentation dans le jeune âge, depuis la mise à l'herbe de tous les animaux à 4 mois jusqu'à 14 mois. Deux niveaux de croissance sont comparés, un niveau bas (NB) et un niveau élevé $(\mathrm{NH})$.

Le traitement NB correspond à une croissance d'environ $900-1000 \mathrm{~g} / \mathrm{j}$ et le traitement $\mathrm{NH}$ à une croissance élevée de $1100 \mathrm{~g} / \mathrm{j}$.

Les animaux sont allotés juste avant la mise à l'herbe vers 4 mois.

\section{Essai 3}

L'essai porte sur le niveau de croissance au cours du second hiver des animaux correspondant aux âges de 21 à 26 mois.

Proches de l'entretien pour les lots NB, les apports alimentaires permettent environ $600 \mathrm{~g}$ de gain moyen quotidien pour le lot $\mathrm{NH}$. Au cours de l'hiver correspondant aux âges de 9 à 14 mois les animaux avaient été alimentés pour un objectif de croissance de l'ordre de $1000 \mathrm{~g} / \mathrm{j}$.

Les animaux sont allotés à la rentrée en stabulation vers 21 mois.

\section{Essai 4}

Le facteur étudié est la vitesse de croissance en stabulation durant les 2 hivers où les animaux sont âgés de 9 à 14 mois et de 21 à 26 mois.

De 9 à 14 mois, les objectifs de croissance quotidienne sont de l'ordre de $800 \mathrm{~g} / \mathrm{j}$ pour les lots NB et de $1000 \mathrm{~g} / \mathrm{j}$ pour les lots $\mathrm{NH}$. Les animaux allotés vers 9 mois restent dans le même traitement durant les 2 hivers. Entre 21 et 26 mois, le niveau alimentaire permet une croissance légèrement positive pour le lot NB et de l'ordre de $600 \mathrm{~g} / \mathrm{j}$ pour le lot $\mathrm{NH}$.

L'essai a été répété sur 2 séries.

\section{Animaux}

Les animaux (tableau I) sont des mâles de race laitière (Normands et Pie-noir) nés de janvier à mars.

Sevrés entre 100 et $110 \mathrm{~kg}$ à un âge de 3 mois environ, les veaux reçoivent du foin et $2 \mathrm{~kg}$ de concentré avant la mise à l'herbe début juin et sont castrés durant l'hiver suivant leur naissance.

\section{Alimentation}

\section{Pâturage}

Entre 4 et 9 mois, les jeunes animaux qui pâturent disposent de repousses de prairies de ray-grass anglais et de trèfle blanc ensilées précocement début mai. Les animaux plus âgés (> 14 mois) pâturent des prairies permanentes ou semées pérennes composées pour moitié de bonnes Graminées (ray-grass ; fétuque ; fléole ; pâturin) associées, notamment, à de l'agrostis et du trèfle blanc. La période de pâturage est un peu supérieure à 200 j, du mois d'avril au mois de novembre.

La conduite du pâturage pratiquée a été décrite par Béranger et Micol (1981). Elle consiste en un pâturage tournant sur 6 à 8 parcelles au cours de 5 à 6 cycles de pâturage. Le nombre de parcelles et le temps de présence des animaux sont ajustés à la pousse de l'herbe, alors que les excédents d'herbe sont fauchés, essentiellement pour être ensilés. Les apports d'azote sont, en moyenne, de 150-200 unités par hectare sur les prairies permanentes et de 200-250 unités sur les prairies temporaires, répartis en 5 à 6 apports successifs au cours de la saison de pâturage.

\section{Stabulation}

Les types de régime hivernaux ainsi que leur complémentation sont décrits dans le tableau I. Les animaux sont logés en groupe dans une stabulation libre disposant d'une aire paillée.

\section{Été 4-9 mois}

Durant cette période, les animaux du traitement restant en stabulation dans l'essai 1 reçoivent une alimentation à base d'ensilage d'herbe pour la série 1 alors que ceux de la série 2 reçoivent à l'auge de l'herbe verte coupée provenant de parcelles équivalentes à celles affectées aux animaux pâturant.

\section{Hiver 9-14 mois}

Les fourrages utilisés sont des ensilages d'herbe, éventuellement de maïs. Du fait de la valeur alimentaire des fourrages et des objectifs de crois- 
sance, la distribution est le plus souvent non limitée et associée à une complémentation adaptée.

\section{Hiver 21-26 mois}

Les niveaux de croissance étant volontairement limités, les animaux reçoivent un fourrage de qualité moyenne à faible (ensilage d'herbe ; foin ou paille) distribué, selon sa valeur alimentaire et les objectifs expérimentaux, de façon limitée ou libérale.

\section{Valeur alimentaire des fourrages}

Les valeurs alimentaires des fourrages sont déterminés à la récolte et en cours d'utilisation par les animaux (tableau II). Les critères mesurés sont les teneurs en matière organique (MO), en matières azotées totales (MAT) et en cellulose brute (CB).

La digestibilité des foins et ensilages a été mesurée, au cours de la période de distribution, sur 6 moutons castrés maintenus en cage à bilan et alimentés à volonté.

La valeur alimentaire des fourrages a été déterminée, selon le système français, en UFL pour l'énergie et en $\mathrm{g}$ de PDIE et PDIN pour l'azote (INRA, 1978, 1988 ; Andrieu et Demarquilly, 1987).

\section{Mesures}

\section{Poids vifs}

Les animaux sont pesés toutes les 2 à 3 sem, systématiquement en début d'après-midi. Au pâturage, les pesées ont lieu le deuxième ou troisième $\mathrm{j}$ suivant un changement de parcelle quand les animaux ont un contenu digestif maximum. Des pesées sont effectuées $2 \mathrm{j}$ consécutifs au moment des changements de régime alimentaire.

\section{Caractéristiques d'abattage et composition corporelle}

Les animaux sont abattus en conditions contrôlées afin de mesurer le poids des différents compartiments corporels (éléments du $5^{e}$ quartier, carcasse...). D'autre part la dissection de la $6^{e}$ côte permet d'estimer, à l'aide des équations de régression établies par Robelin et Geay (1975), la composition de la carcasse et du corps entier en tissus adipeux, musculaire et squelettique.

Les animaux sont abattus à un même poids vif moyen d'environ $720 \mathrm{~kg}$ afin de mettre en évidence les effets stricts des traitements sur les caractéristiques d'abattage et corporelles. Au sein d'un lot expérimental, les décisions d'abattage sont prises à partir d'une estimation, par palpation, de l'état d'engraissement des animaux.

\section{Traitement des données et analyse statistique}

Les courbes de croissance individuelles ont été élaborées pour chaque période de la vie de l'animal par régression polynomiale à partir des pesées réalisées.

Une période finit le jour de la mise à l'herbe ou de la rentrée en stabulation des animaux et débute après 15 j de transition alimentaire. L'écart de poids en transition est estimé par la variation de poids au cours de ces $15 \mathrm{j}$.

Les données sur les vitesses de croissance, les caractéristiques d'abattage et de composition corporelle ont été analysées avec le logiciel statistique SAS (1980). Les procédures utilisées ont été General Linear Model ou Anova pour les analyses de variance avec ou sans covariable(s); Univariate pour les tests de normalité de Ttest pour les comparaisons entre 2 lots expérimentaux.

\section{RÉSULTATS}

Les résultats sont présentés selon les facteurs étudiés dans les tableaux III, IV et V.

\section{Essai 1 : effet du mode de conduite avant 9 mois et nature du régime hivernal}

\section{Mode de conduite avant 9 mois}

Peu de différences notables apparaissent entre les lots STA alimentés à l'auge, entre 3 et 9 mois, soit avec de l'ensilage d'herbe soit avec de l'herbe coupée. Aussi, par la 
Tableau II. Composition chimique et valeur nutritive des aliments utilisés pendant les phases hivernales.

MS MM MAT CB CUD UFL PDIN PDIE (\%) (\%MS) (\%MS) (\%MS) MO (/kg MS) (/kg MS) (/kg MS)

Hiver 9-14 mois

Ensilages d'herbe

Série 1

Série 2

Série 3

Série 4

Série 5

Série 6

Ensilages de maïs

Série 1

Série 2

Série 3

Série 4

Série 6

$\begin{array}{rrrrrrrr}21,4 & 8,1 & 14,8 & 26,3 & 75,0 & 0,94 & 86 & 71 \\ 21,4 & 11,1 & 10,9 & 29,8 & 59,0 & 0,64 & 64 & 57 \\ 19,2 & 8,0 & 12,8 & 26,0 & 75,5 & 0,94 & 77 & 82 \\ 21,2 & 12,0 & 17,8 & 24,5 & & 0,87 & 105 & 109 \\ 21,0 & 9,0 & 12,8 & 28,9 & 73,6 & 0,81 & 75 & 78 \\ 27,8 & 15,0 & 8,5 & 28,3 & 66,7 & 0,68 & 51 & 65\end{array}$

$\begin{array}{llllllll}22,9 & 5,8 & 8,3 & 28,0 & 63,7 & 0,77 & 49 & 57\end{array}$

$\begin{array}{llllllll}27,6 & 4,9 & 7,9 & 20,1 & 69,7 & 0,89 & 46 & 60\end{array}$

$\begin{array}{llllllll}25,3 & 4,0 & 8,1 & 20,3 & 69,9 & 0,90 & 40 & 64\end{array}$

$\begin{array}{llllllll}23,5 & 5,0 & 8,4 & 21,5 & 66,2 & 0,77 & 49 & 59\end{array}$

$\begin{array}{llllllll}25,1 & 5,3 & 8,3 & 23,0 & 70,6 & 0,84 & 49 & 63\end{array}$

Hiver 21-26 mois

Ensilages d'herbe

Série 1

Série 2

Série 3

Série 4

Série 5

Série 6

Foins

Série 1

Série 2

Série 3

Paille

$\begin{array}{rrrrrrrr}24,1 & 10,8 & 8,9 & 30,5 & 54,5 & 0,58 & 54 & 76 \\ 24,3 & 11,6 & 11,3 & 29,4 & 65,8 & 0,74 & 66 & 70 \\ 24,7 & 13,0 & 8,5 & 32,0 & 57,7 & 0,61 & 51 & 67 \\ 24,7 & 11,2 & 11,3 & 27,2 & 66,6 & 0,72 & 68 & 76 \\ 23,4 & 10,6 & 10,5 & 30,0 & 68,6 & 0,73 & 63 & 71 \\ 24,6 & 8,1 & 10,5 & 34,2 & 61,3 & 0,64 & 62 & 63\end{array}$

$\begin{array}{llllllll}85,0 & 7,4 & 8,0 & 32,0 & 55,2 & 0,62 & 50 & 64\end{array}$

$\begin{array}{llllllll}85,0 & 7,2 & 9,0 & 31,4 & 59,8 & 0,67 & 56 & 69\end{array}$

$\begin{array}{llllllll}85,0 & 7,1 & 7,5 & 32,5 & 54,0 & 0,60 & 56 & 62\end{array}$

$\begin{array}{llllll}82,0 & 8,6 & 1,5 & 42,9 & 0,41 & 10\end{array}$

Concentrés

Tourteau de colza

Concentré commercial pour les séries 5 et 6

Concentré commercial pour les séries 1, 2 et 3

Orge

Pulpe déshydratée

Composé azoté liquide (vinasse)

$\begin{array}{rrrrrrr}88,0 & 7,1 & 36,9 & 11,9 & 0,95 & 247 & 183 \\ 86,0 & & & & 1,10 & 147 & 138 \\ & & & & & & \\ 86,0 & & & & 0,95 & 115 & 110 \\ 86,0 & 2,5 & 12,1 & 6,0 & 1,16 & 84 & 105 \\ 86,1 & 10,3 & 11,2 & 17,9 & 1,00 & 70 & 98 \\ 70,0 & & 60,5 & & 1,00 & 367 & 158\end{array}$

MS : matières sèches ; MM : matières minérales ; MAT : matières azotées totales ; CB : cellulose brute ; MO : matière organique ; CUD : coefficient d'utilisation digestive ; UFL : unité fourragère lait ; PDIN : protéines digestibles dans l'intestin grêle selon l'azote; PDIE : protéines digestibles dans l'intestin grêle selon l'énergie. 


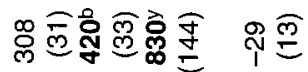

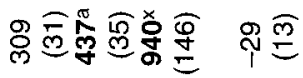

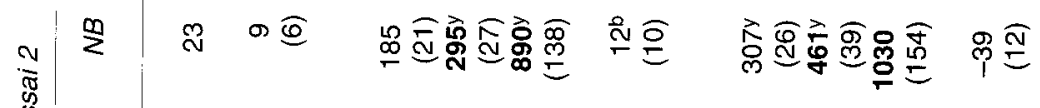

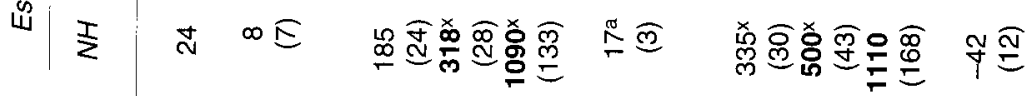

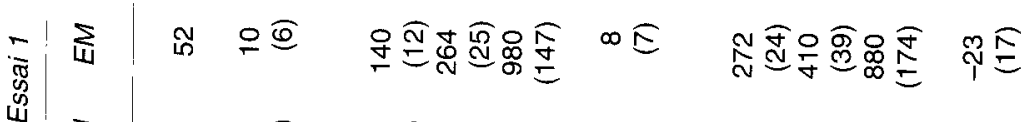

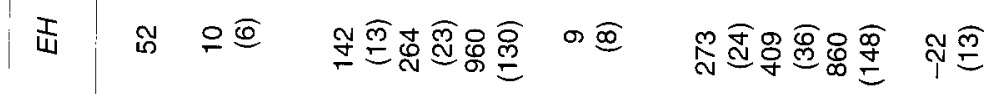

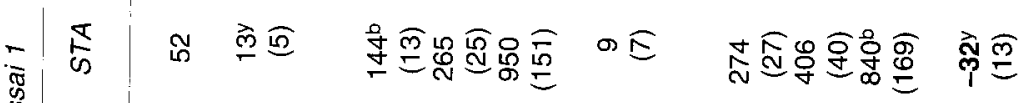

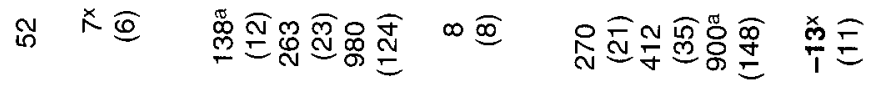

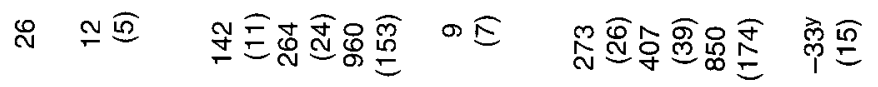

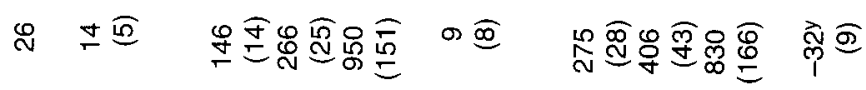

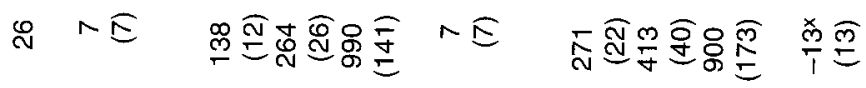

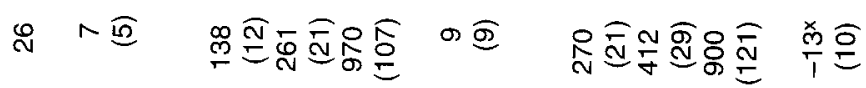

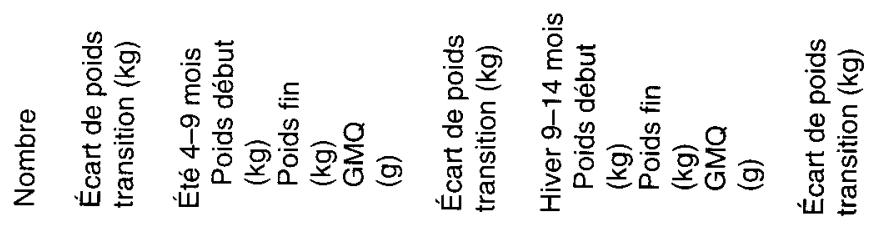




\begin{tabular}{|c|c|c|}
\hline 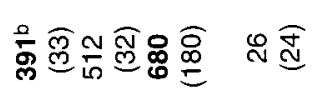 & 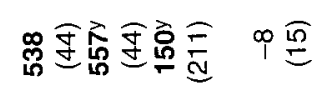 & 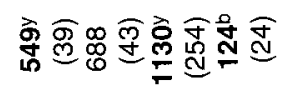 \\
\hline \multirow[t]{3}{*}{ 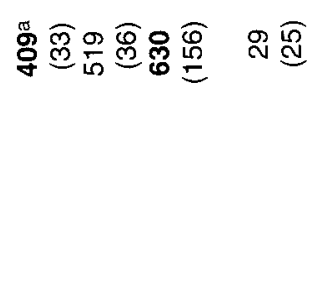 } & 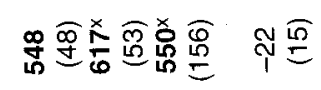 & 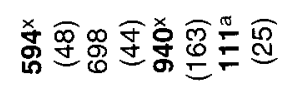 \\
\hline & 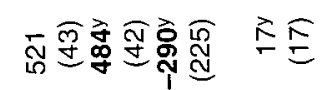 & 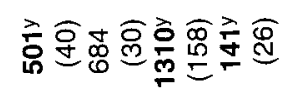 \\
\hline & 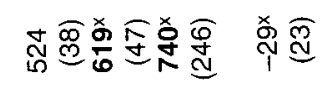 & 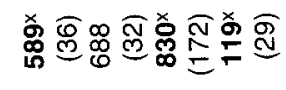 \\
\hline
\end{tabular}

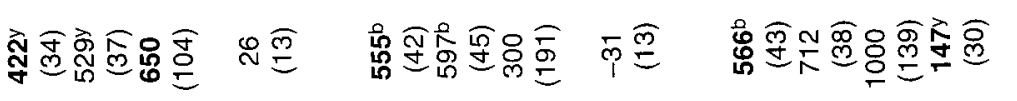

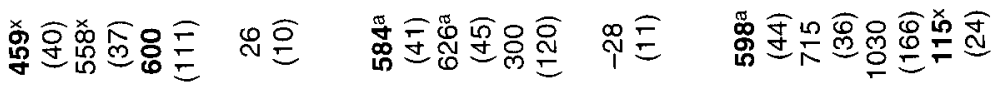

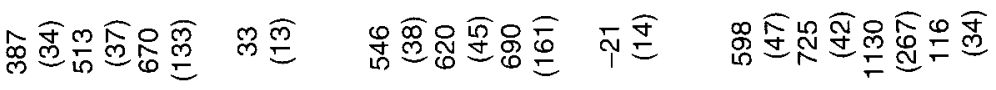

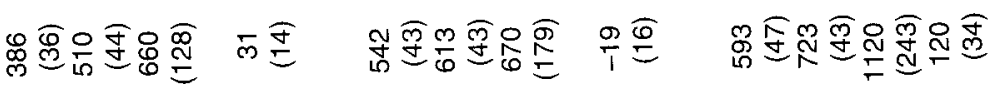

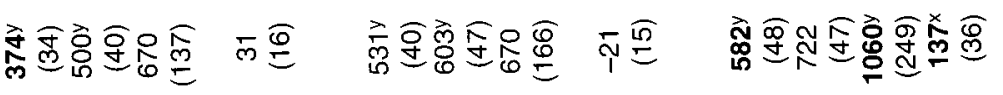

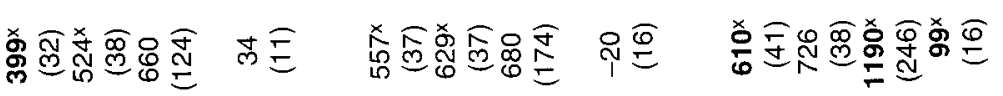

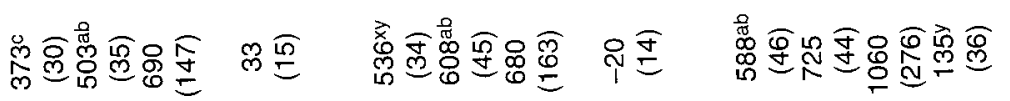

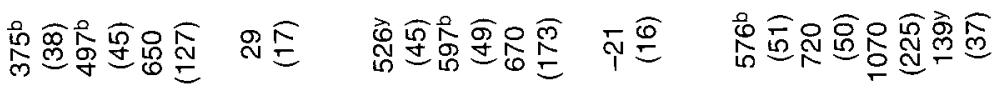

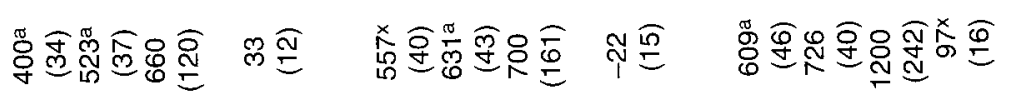

$\ddot{a}$

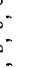

.

2

:-

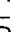

v

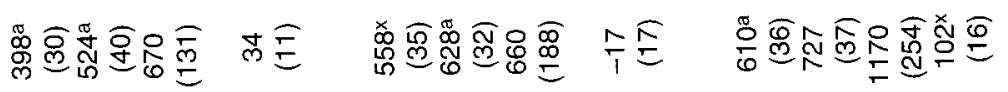




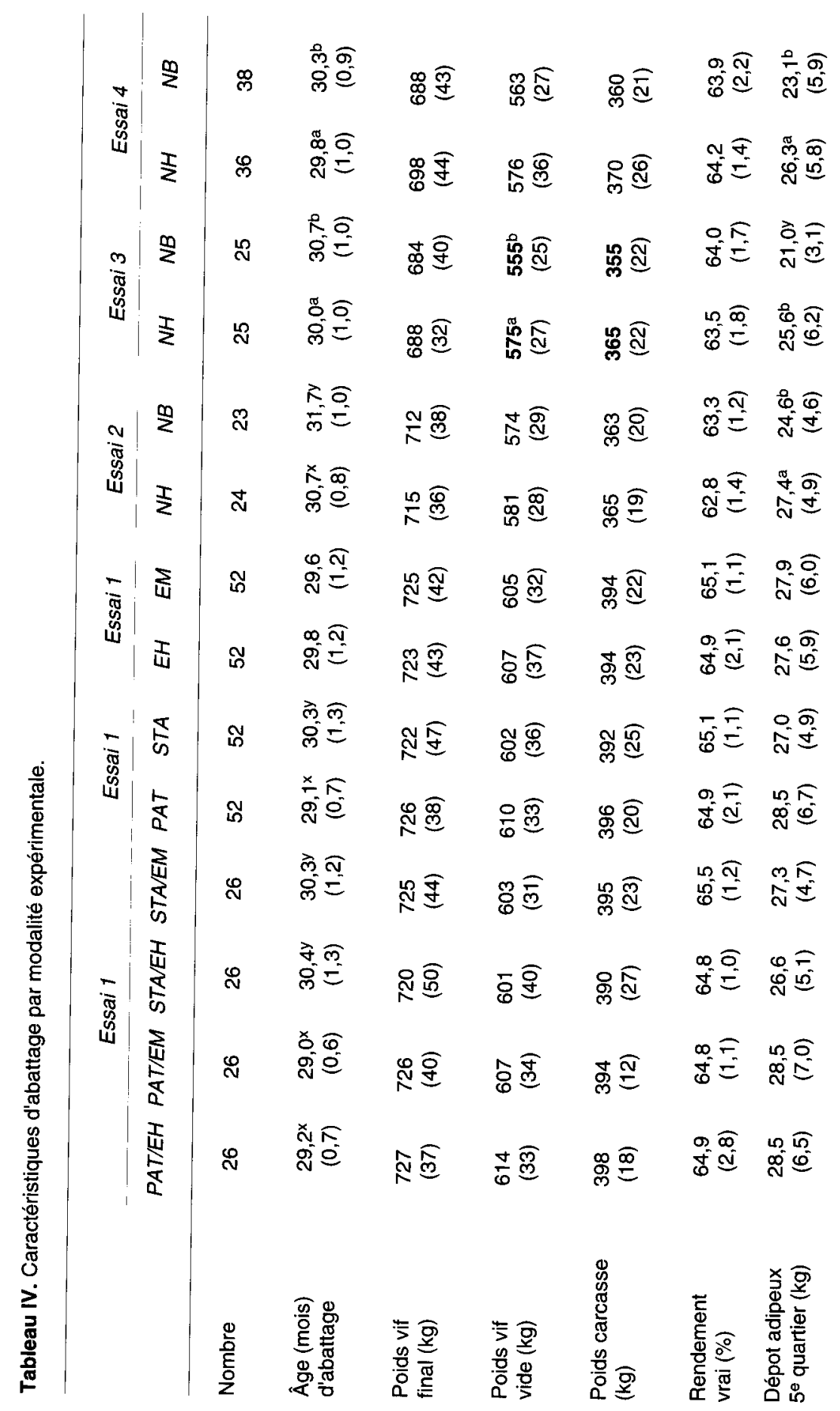




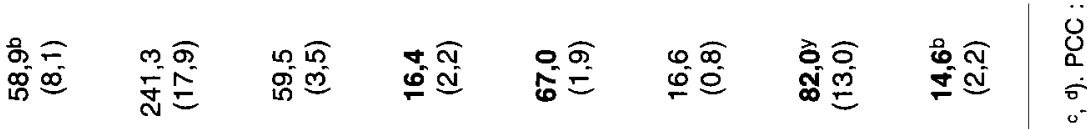

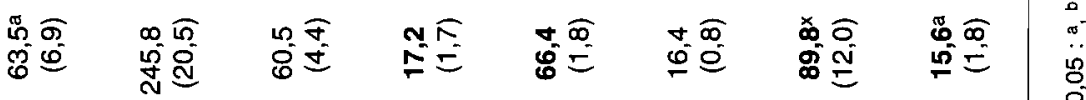

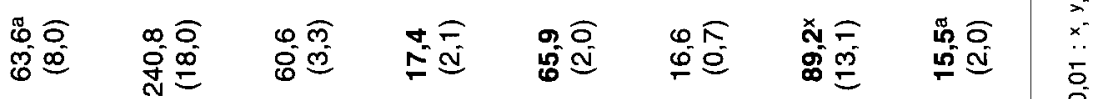

듯ำ

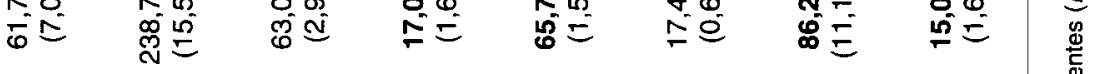

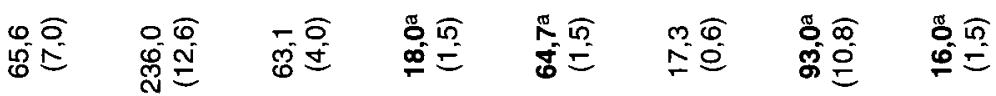

m

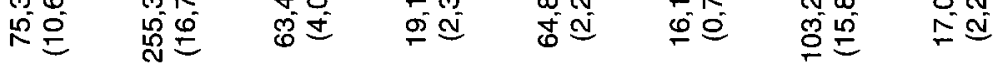

$\infty$ 으

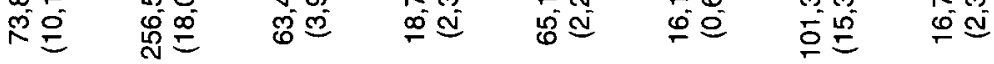

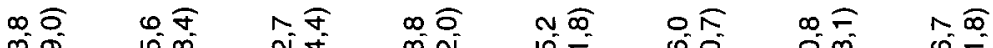

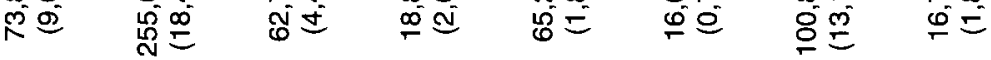

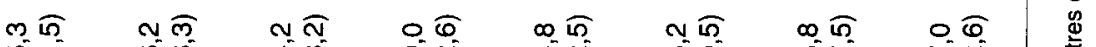

舟E

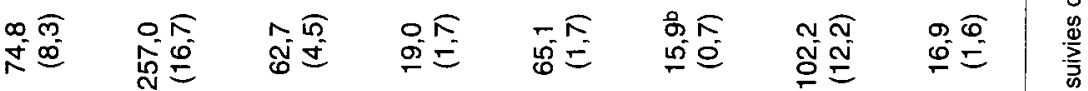

$\infty$ ก ก

ND 岇

$\infty \widehat{0} 0$ -

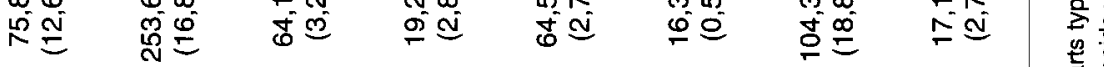

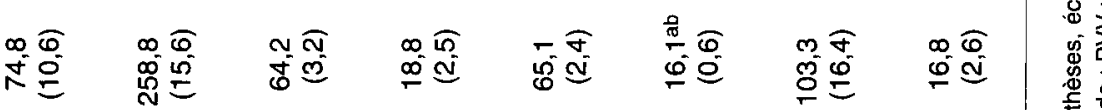

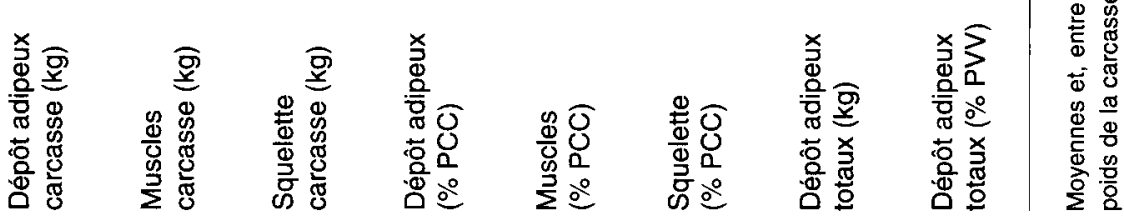




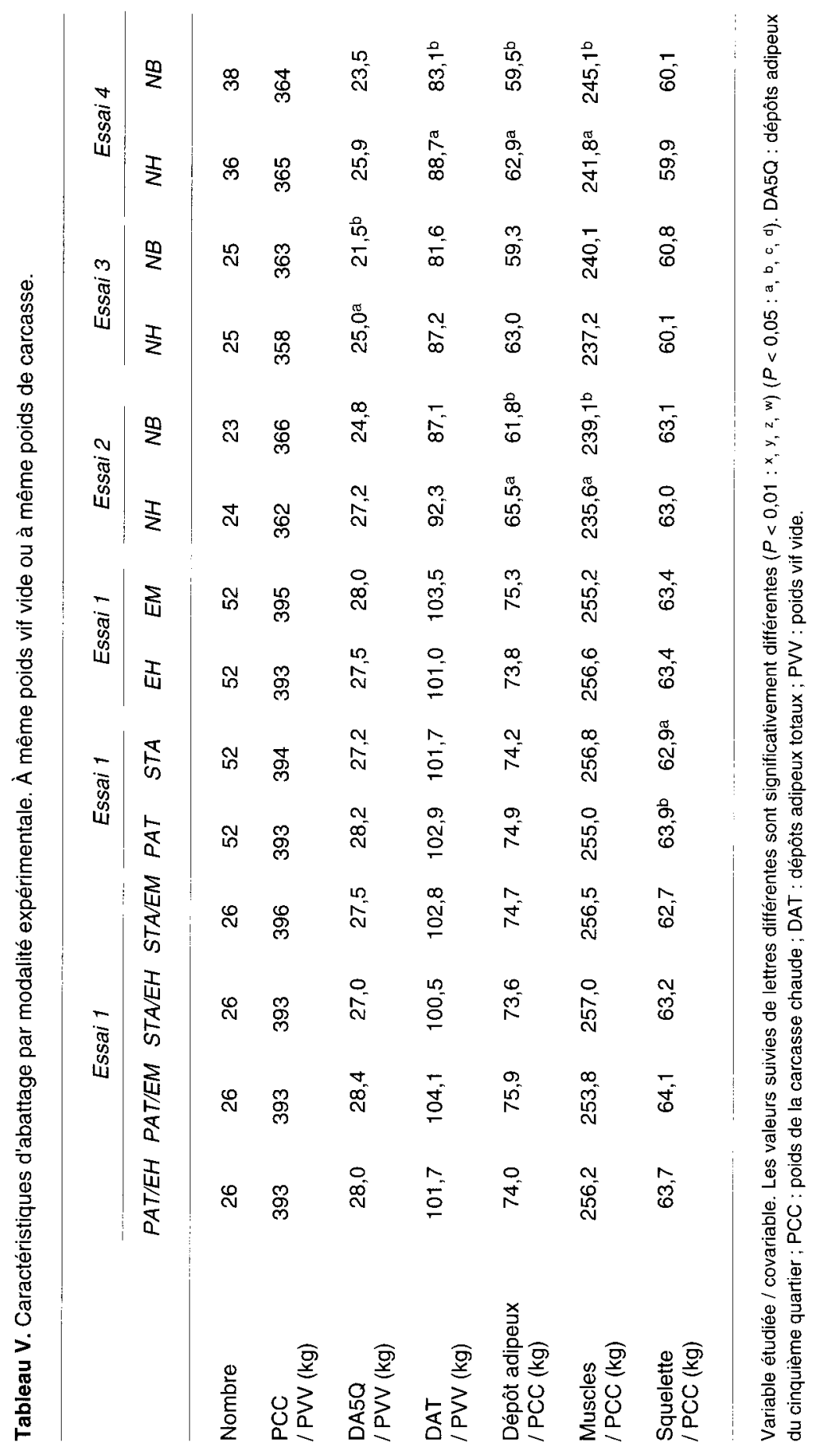


suite les résultats des 2 séries seront confondus et présentés en une seule modalité du traitement STA. Les animaux mis à l'herbe (PAT) ont un léger retard de poids (6 $\mathrm{kg}$ ) durant la période de transition en stabulation, retard qui est rattrapé à 9 mois (263 kg contre $265 \mathrm{~kg}$ ).

À la seconde mise à l'herbe, à 14 mois, les lots ont un poids voisin, mais, durant la transition de la mise à l'herbe, un net avantage est acquis par le lot PAT qui perd seulement $13 \mathrm{~kg}$ contre $32 \mathrm{~kg}$ pour le lot STA (fig 1). En outre, la perte de poids du lot STA est plus importante pour les animaux qui ont reçu de l'ensilage d'herbe (série 1) que pour ceux qui ont reçu de l'herbe coupée (série 2) durant la période de 5 à 9 mois. Tout au long de la saison de pâturage de 14 à 21 mois, les croissances sont identiques (660 g/j pour le lot PAT contre $670 \mathrm{~g} / \mathrm{j}$ pour le lot STA). L'avantage acquis $(25 \mathrm{~kg})$ à 15 mois est conservé jusqu'à l'abattage, le traitement STA nécessitant 38 j supplémentaires de finition pour atteindre un poids vif identique de $724 \mathrm{~kg}$. La croissance au pâturage diminuant au cours de la saison, la durée de finition plus longue du traitement STA explique la baisse de la croissance moyenne de ce lot.
En ce qui concerne les caractéristiques d'abattage, aucune différence entre traitements n'apparaît. Les carcasses atteignent en moyenne $394 \mathrm{~kg}$ et contiennent $65 \%$ de muscles et $19,9 \%$ de dépôts adipeux.

\section{Type de régime hivernal}

La nature du régime du premier hiver (ensilage d'herbe ou de maïs) n'a aucun effet sur les performances de croissance ou sur les caractéristiques d'abattage. De même, aucune interaction du type de régime avec le facteur mode de conduite avant 9 mois n'a été mise en évidence.

Toutefois, au sein de chaque série, les pertes de poids après la mise à l'herbe à 14 mois sont significativement différentes $(P<0,01)$ entre type de régime hivernal. Pour la série 1 la perte de poids est supérieure pour le traitement EM $(-32 \mathrm{vs}-19 \mathrm{~kg})$ alors que, dans le cas de la série 2, le résultat est inversé, le traitement EH perdant 25 vs $14 \mathrm{~kg}$ pour le traitement EM. Ceci s'explique par des différences de digestibilité entre fourrages (tableau II), les lots recevant le fourrage le plus digestible (ensilage de maïs pour la série 1 et ensilage d'herbe pour
Fig 1. Effet du mode d'alimentation entre 4 et 9 mois sur la croissance (bœufs de l'essai 1).

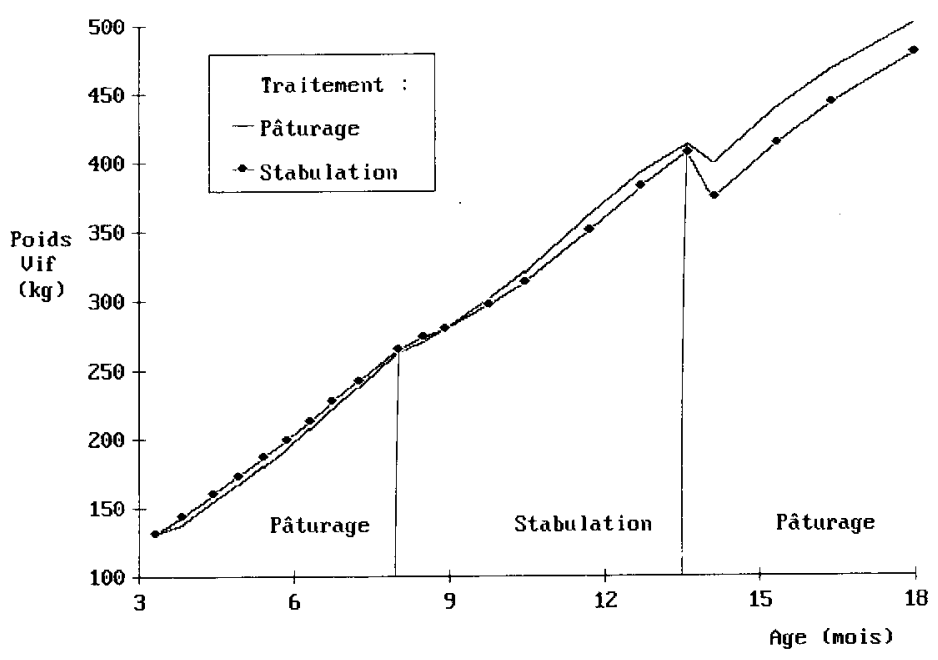


la série 2) perdant moins de poids de contenu digestif à la mise à l'herbe.

\section{Essai 2 : effet du niveau de croissance dans le jeune âge}

L'augmentation du niveau alimentaire entre 4 et 14 mois a entraîné une différence significative de croissance ( $+37 \mathrm{~kg}$ après la mise à l'herbe à 14 mois). Lors de l'été suivant le traitement, le lot NB a une croissance non significativement supérieure au traitement $\mathrm{NH}$ (650 vs $600 \mathrm{~g} / \mathrm{j}$ ), mais ce qui permet de réduire l'écart initial à $29 \mathrm{~kg}$ en fin de période de pâturage. Cet écart subsiste jusqu'à la période de finition et, pour obtenir un même poids d'abattage de $713 \mathrm{~kg}, 32 \mathrm{j}$ supplémentaires ont été nécessaires au traitement NB.

Les $364 \mathrm{~kg}$ de carcasses obtenus contiennent $65,2 \%$ de muscles, $17,5 \%$ de tissus adipeux et $17,3 \%$ de squelette. Les animaux du lot NB sont moins gras ( -1 point de tissus adipeux dans la carcasse ; $P<0,05)$ et ont déposé plus de muscles (+0,9 point ; $P<0,05$ ).

\section{Essai 3 : effet du niveau de croissance au cours de I'hiver entre 21 et 26 mois d'âge}

Les croissances durant le second hiver entre 21 et 26 mois (fig 2) ont été très différentes (écart de $1030 \mathrm{~g} / \mathrm{j}$ ). À la mise à l'herbe, le poids des animaux diffère de $135 \mathrm{~kg}(P<0,01)$ entre les 2 traitements. Au cours de la transition, le lot NB compense une partie de son retard $(+17$ vs $-29 \mathrm{~kg}$ de perte de poids ; $P<0,01$ ). Ensuite, la croissance au pâturage est significativement supérieure pour le traitement NB (1 $310 \mathrm{~g} / \mathrm{j}$ contre $830 \mathrm{~g} / \mathrm{j} ; P<0,01)$. Malgré cela, il leur faut 22 j supplémentaires de finition $(P<0,01)$ pour être abattus à même poids vif.

Si, dans le traitement NB, le poids de carcasse est plus faible (355 kg contre $365 \mathrm{~kg}$ ), en revanche l'état d'engraissement est significativement moindre : 14,3 vs $15,5 \%$ de dépôts adipeux dans la masse corporelle $(P<0,05)$. En revanche la composition de la carcasse n'est pas significativement différente.

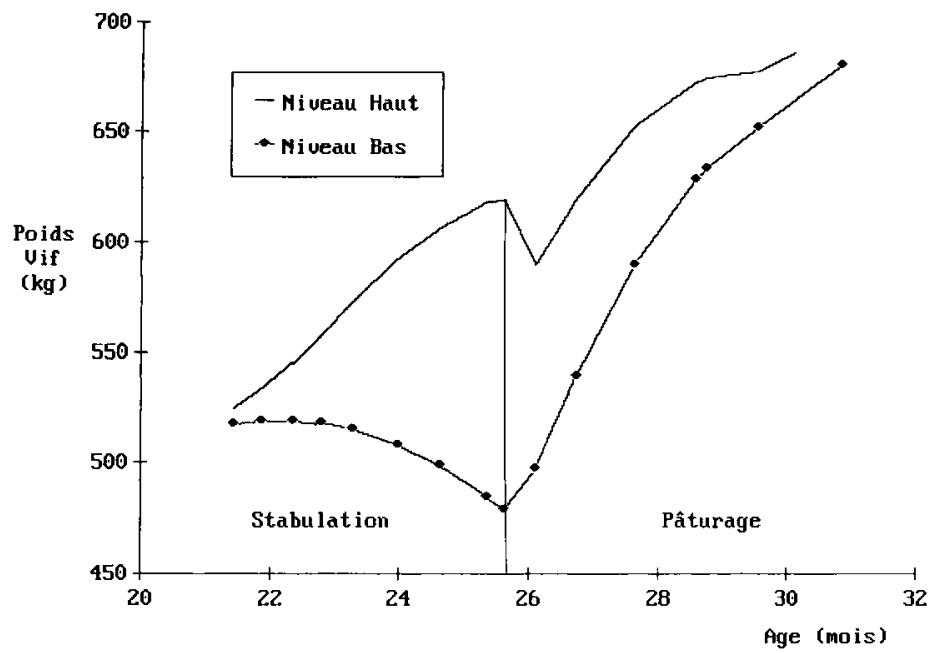

Fig 2. Effet du niveau alimentaire sur la croissance entre 21 mois et l'abattage (bœufs de l'essai 3). 
Essai 4 : effet du niveau de croissance au cours des hivers entre 9 et 14 mois et entre 21 et 26 mois

Les 2 séries ayant abouti à des résultats similaires : ceux-ci sont agrégés pour ne présenter que les modalités NH et NB.

\section{Niveaux de croissance entre 9 et 14 mois (fig 3)}

Grâce à une croissance hivernale supérieure de $110 \mathrm{~g} / \mathrm{j}$, les animaux du traitement $\mathrm{NH}$ sont plus lourds à la mise à l'herbe de $17 \mathrm{~kg}$. Au cours des 178 j du pâturage sujvant, la croissance des lots NB légèrement supérieure $(+50 \mathrm{~g} / \mathrm{j})$ ne permet plus de dissocier les 2 traitements en début de stabulation à 21 mois $(543 \mathrm{~kg})$.

\section{Niveaux de croissance entre 21 et 26 mois}

La restriction alimentaire sévère du lot NB a permis de creuser d'importants écarts de croissance, $550 \mathrm{~g} / \mathrm{j}$ pour $\mathrm{NH}$ vs $150 \mathrm{~g} / \mathrm{j}$ pour NB qui est mis à l'herbe avec un poids inférieur de $60 \mathrm{~kg}$ à celui du traitement $\mathrm{NH}$. $\mathrm{Au}$ cours de la transition, le lot NB perd sensiblement moins de contenu digestif ( -8 vs $-22 \mathrm{~kg}$ pour le lot $\mathrm{NH}$ ).

À l'issue de la période de finition à l'herbe, les animaux sont abattus sensiblement au même poids (698 vs $688 \mathrm{~kg}$ ) par suite d'une croissance plus élevée (1 130 vs $940 \mathrm{~g} / \mathrm{j}, P<0,01$ ) et d'une durée de finition plus longue (125 vs $11 \mathrm{j}$ ) du lot NB.

Les animaux ont un poids de carcasse de $365 \mathrm{~kg}$, contenant $66,7 \%$ de muscles, $16,8 \%$ de dépôts adipeux et $16,5 \%$ de squelette. Les individus du traitement NB sont moins gras et déposent plus de muscles. Cet écart s'explique en partie par la différence de poids à l'abattage. Mais, ramenées à même masse corporelle ou à même poids de carcasse, les différences restent significatives : $-3 \mathrm{~kg}$ de dépôts adipeux intermusculaires et $+3 \mathrm{~kg}$ de muscles à même poids de carcasse pour le traitement NB $(P<0,05)$.
Fig 3. Effet du niveau alimentaire sur la croissance entre 9 et 21 mois (bœufs de l'essai 5).

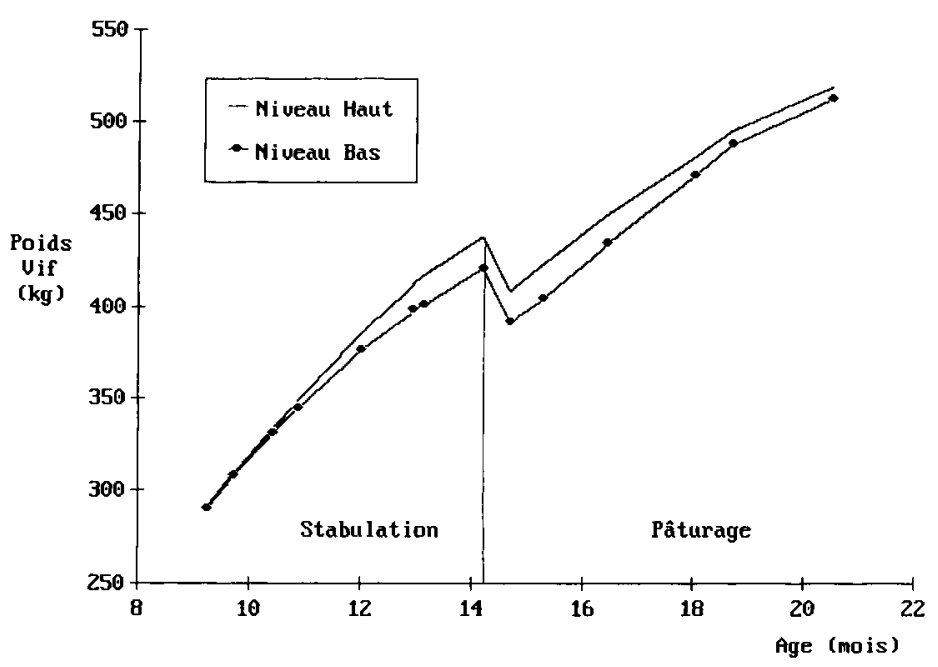




\section{DISCUSSION}

\section{La pratique de la pâture}

Le pâturage pratiqué à un âge précoce, entre 5 et 9 mois, permet aux animaux de mieux valoriser les ressources en herbe au cours des périodes ultérieures de pâturage. En effet, si tous les lots d'animaux perdent du poids à la mise à l'herbe à 14 mois, les animaux des lots PAT en perdent moins $(39 \%$ des pertes de poids du traitement STA) et cet avantage se maintient par la suite.

La mise à l'herbe provoque des changements rapides de contenu digestif dont le poids diminue en quelques jours, l'herbe étant plus digestible que les fourrages hivernaux.

II ressort de divers travaux (Wilson, 1960 ; Wilson et Osbourn, 1960) que le développement du tractus digestif est surtout lié à l'âge. Ainsi, le type de régime après sevrage ne semble pas provoquer de variation dans le développement du tractus digestif. En conséquence, la différence de perte de poids à la mise à l'herbe à 14 mois ne doit pas provenir d'évolutions différentes du tractus digestif, les animaux ayant été conduits selon un même niveau de croissance.

L'écart observé est certainement le fruit d'une accoutumance plus rapide au pâturage pour les animaux du traitement PAT. Cela peut être le fait d'une meilleure adaptation digestive et métabolique, mais aussi de consommations supérieures au pâturage comme l'ont constaté Le Du et al (1976) et Arnold (1970) pour des bovins et des ovins.

La rapidité d'adaptation des animaux du traitement PAT lors du pâturage à 14 mois ne s'accompagne pas de croissances ultérieures supérieures, ce qui va à l'encontre de résultats obtenus dans 2 essais par l'Institut technique de l'élevage bovin (ITEB, 1984, 1986). Ces 2 essais, réalisés avec des bœufs Normands, ne montraient pas de dif- férences de perte de poids à la mise à l'herbe accompagnée d'une transition alimentaire. En revanche, le niveau de croissance durant la saison de pâturage des animaux ayant déjà pâturé à un âge précoce était supérieur de 100 à $200 \mathrm{~g} / \mathrm{j}$. Une explication de la similitude des croissances de nos animaux au pâturage pourrait venir des conditions parasitaires. En effet, dans nos conditions, les animaux n'ont pas eu à souffrir d'infestations parasitaires. Dans des circonstances plus favorables aux parasites, peut-être aurions nous vu un avantage en faveur des animaux ayant déjà la pratique de la pâture.

Même en l'absence de parasitisme, la pratique précoce de la pâture a cependant permis aux animaux un gain de poids de $5 \%$ à la rentrée en stabulation vers 21 mois (524 vs $500 \mathrm{~kg}$ ).

\section{La croissance compensatrice}

Tous les lots d'animaux ayant réalisé des croîts inférieurs suite à un moindre niveau alimentaire ont ensuite compensé en partie lors de la période d'alimentation libérale.

Les lots $\mathrm{NH}$ étant considérés comme témoins, des niveaux de croissance limités, voire négatifs, lors de l'hiver entre 21 et 26 mois, se traduisent par des croissances nettement supérieures au pâturage. Le taux de compensation est défini (par rapport au lot témoin) comme étant la croissance supplémentaire faisant suite à une croissance inférieure de $100 \mathrm{~g}$ lors de la période précédente. Ce taux est identique - $47 \%-$ dans les essais 3 et 4 . La faible durée, de l'ordre de 100 à $150 \mathrm{j}$, de la période d'alimentation libérale à l'herbe qui fait suite à la période d'alimentation hivernale restreinte nécessite un allongement significatif de la finition pour obtenir une compensation complète et un abattage à même poids. Dans les 2 essais, malgré des écarts de crois- 
sance entre les traitements NH et NB très différents, le taux de compensation est le même. Ceci nuance les conclusions de Ryan (1990) selon lequel l'intensité de la restriction ne joue pas sur l'ampleur de la compensation. Dans l'essai 3, la sévérité de la restriction (les animaux ont perdu du poids entre 21 et 26 mois) explique la croissance très élevée des animaux du traitement NB au pâturage à l'inverse des animaux du lot NH du même essai qui ont eu les croissances les plus faibles de tous les lots en finition.

Entre 14 et 21 mois, les taux de compensation très variables (respectivement 63 et $45 \%$ pour les essais 2 et 4 ) restent inférieurs à $100 \%$. Malgré une période de pâturage plus longue que l'hiver qui le précédait (178 vs 136 j), les écarts creusés au cours de l'hiver entre 9 et 14 mois ne sont que partiellement comblés à la rentrée en stabulation vers 21 mois. Ainsi, dans l'essai 4, l'écart de poids à la mise à l'herbe à 14 mois est comblé à $59 \%$ à la rentrée en stabulation à 21 mois, ce qui est en accord avec les valeurs recueillies par O'Donovan (1984) et observées plus récemment par Troccon (1993) sur des génisses de type Pie noire. Toutefois, si les écarts de poids subsistent, ils ne sont plus significatifs comme l'avait déjà montré Micol (1986) lors d'essais antérieurs où des croissances hivernales de 640 vs $1040 \mathrm{~g} / \mathrm{j}$ avaient été presque entièrement compensées au cours de la saison de pâturage suivante.

En revanche, une croissance plus élevée entre 5 et 14 mois assure un avantage qui semble acquis. Ainsi, dans l'essai 2, l'essentiel de l'écart est réalisé précocement au pâturage, entre 5 et 9 mois. L'augmentation du niveau de croissance est alors surtout le fait d'une meilleure régularité de celleci, les animaux du lot NB réagissant plus fortement aux aléas alimentaires (diminution de la qualité de l'herbe en automne, rentrée en stabulation, qualités variables des ensilages hivernaux).
Enfin dans tous les essais concernés, les animaux des lots $\mathrm{NH}$ ont réalisé des croissances supérieures résultant de niveaux alimentaires plus élevés. Cette différence permet de réduire la durée de finition au pâturage de 13 à 32 j (1 à $3 \%$ de la durée de vie). De plus elle s'accompagne d'un changement de composition corporelle, les animaux des lots $\mathrm{NH}$ ayant systématiquement plus de dépôts adipeux dans la masse corporelle comme dans la carcasse.

\section{CONCLUSION ET RECOMMANDATIONS}

Les 275 bœufs Normands et Pie Noirs ont été abattus à l'âge de 30 mois à un poids vif moyen de $707 \mathrm{~kg} \pm 43$. Ils ont produit des carcasses pesant en moyenne $375 \mathrm{~kg} \pm 27$ et comprenant $65,7 \% \pm 2,1$ de muscles et $17,7 \% \pm 2,2$ de tissus adipeux.

Ces résultats varient selon les modalités d'élevage testées (33 kg d'écart entre les poids de carcasses moyens des lots extrêmes). Ainsi, la pratique précoce de la pâture vers 5 mois améliore les performances de production. En revanche, la comparaison de plusieurs régimes hivernaux entre 9 et 14 mois montre que, conduits à même croissance, les animaux ne présentent pas de différences ultérieures de croissance et de caractéristiques d'abattage.

Les fluctuations de croissance provoquées ont eu des effets différents selon la période d'application. Comme cela a été établi dans de nombreux travaux, nous confirmons que la croissance compensatrice s'exprime peu avant 9 mois, âge avant lequel tout retard dans la croissance est donc à éviter. Un niveau de croissance élevé permet alors d'avancer la date d'abattage.

Entre 9 et 14 mois, les niveaux de croissance peuvent se situer entre 650 et 1000 $\mathrm{g} / \mathrm{j}$. Dans ces limites, la réduction des croissances hivernales se traduira par une crois- 
sance compensatrice presque totale durant la période du pâturage suivante.

Durant le dernier hiver les croissances peuvent être très faibles, voire nulles, l'obtention d'un poids suffisant à l'abattage nécessitera simplement quelques jours de finition supplémentaires au pâturage.

Le niveau de croissance à un impact sur les caractéristiques d'abattage. Quel que soit l'âge, les animaux ayant eu un niveau plus élevé de croissance, surtout réalisé par des apports d'aliments concentrés, sont, en moyenne, abattus plus gras que leurs homologues non complémentés.

Les restrictions hivernales, de même qu'un niveau de croissance moyen lors des premier et second hivers permettent d'économiser des intrants et d'obtenir une utilisation maximale de l'herbe pâturée. Mais des croissances supérieures, nécessitant des apports supplémentaires, procurent cependant davantage de sécurité pour la finition au pâturage. En effet, en cas de conditions climatiques défavorables, il peut être nécessaire d'apporter un complément d'aliments concentrés à l'herbe aux animaux non finis. Le choix de la croissance hivernale avant la finition à l'herbe est alors un moyen d'agir sur la période de sortie des animaux du pâturage à l'automne suivant. Son intérêt variera selon l'importance accordée à la disponibilité en herbe et aux conditions du marché.

\section{REMERCIEMENTS}

Nous voudrions présenter nos sincères remerciements au personnel du service d'expérimentation animale du domaine qui a participé activement à la réalisation de ces expériences et tout particulièrement à son directeur, A Muller.

\section{RÉFÉRENCES}

Andrieu J, Demarquilly C (1987) Valeur nutritive des fourrages : tables et prévision. Bull Tech CRZV Theix INRA 70, 61-73
Arnold GW (1970) Regulation of food intake by grazing ruminants. In : Physiology of digestion and metabolism in the ruminant (AT Phillipson, ed)

Bèranger C, Micol D (1981) Utilisation de l'herbe par ies bovins au pâturage. Importance du chargement et du mode d'exploitation. Fourrages 85, 73-93

Berge $P$ (1991) Long-term effects of alimentation during calfhood on subsequent performances in beef cattle. Livest Prod' Sci 28, 179-201

Berge P, Geay Y, Micol D (1991) Effect of feeds and growth rate during the growing phase on subsequent performances during the fattening period and carcass composition in Friesian young bulls. Livest Prod Sci $28,201-213$

Giovanni R (1982) Mise à l'herbe après sevrage des veaux laitiers nés en fin d'hiver : influence du niveau d'alimentation avant la mise à l'herbe et de la complémentation énergétique au pâturage. Bull Tech CRZV Theix INRA 50, 33-45

INRA (1978) Alimentation des ruminants. Éd INRA Publications, $598 \mathrm{p}$

ITEB (1984) Bœufs Normands de 30 mois nès en fin d'hiver et finis au pâturage. $\mathrm{N}^{\circ} 84033$

ITEB (1986) Bœuts Normands finis à l'auge à 26 mois ou au pâturage à 30 mois. $N^{\circ} 86032$

INRA (1988) Alimentation des bovins, ovins et caprins (R Jarrige, ed), INRA, Paris, $476 p$

Le Du YLP, Baker RD, Barker JM (1976) Milk-fed calves: the effect of length of milk feeding period and milk intake upon herbage intake and performance of grazing calves. J Agric Sci (Camb) 87, 197-204

Micol D (1986) Production de viande de bœufs et de jeunes taureaux. $I n$ : Production de viande bovine (D Micol, ed), INRA

O'Donovan PPB (1984) Compensatory gain in cattle and sheep. Nutr Abst Rev, Series B. Livestock Feeds and Feeding 54, 389-410

OFIVAL (1991) Le marché des viandes et des produits avicoles en 1991

Robelin J, Geay Y (1975) Estimation de la composition de la carcasse des taurillons à partir de la composition de la $6^{6}$ cóte. Bull Tech CRZV Theix INRA 22, 41-44

Ryan WJ (1990) Compensatory growth in cattle and sheep (revue). Nutr Abst Rev 60, 653-664

SAS (1980) SAS/STAT User's Guide, Release 6.03 Edition, Cary, NC, États-Unis

Troccon JL (1993) Effects de l'alimentation hivernale des génisses sur les performances et la longévité des vaches. INRA Prod Anim 6, 345-356

Wilson PN (1960) The effect of plane of nutrition on the growth and development of the east African dwarf goat: The effect of plane of nutrition and sex on the carcass composition of the kid at two stages of growth, $16 \mathrm{lb}$ live weight and $30 \mathrm{lb}$ live weight. $J$ Agric Sci $54,105-134$

Wilson PN, Osbourn DF (1960) Compensatory growth after undernutrition in mammals and birds. Biol Rev $35,324-363$ 